ARTICLE

\title{
Structure-based machine-guided mapping of amyloid sequence space reveals uncharted sequence clusters with higher solubilities
}

Nikolaos Louros ${ }^{1,2}$, Gabriele Orlando ${ }^{1,2}$, Matthias De Vleeschouwer ${ }^{1,2}$, Frederic Rousseau (iD ${ }^{1,2 凶}$ \& Joost Schymkowitz (1) ${ }^{1,2 \otimes}$

The amyloid conformation can be adopted by a variety of sequences, but the precise boundaries of amyloid sequence space are still unclear. The currently charted amyloid sequence space is strongly biased towards hydrophobic, beta-sheet prone sequences that form the core of globular proteins and by $\mathrm{Q} / \mathrm{N} / \mathrm{Y}$ rich yeast prions. Here, we took advantage of the increasing amount of high-resolution structural information on amyloid cores currently available in the protein databank to implement a machine learning approach, named Cordax (https://cordax.switchlab.org), that explores amyloid sequence beyond its current boundaries. Clustering by t-Distributed Stochastic Neighbour Embedding (t-SNE) shows how our approach resulted in an expansion away from hydrophobic amyloid sequences towards clusters of lower aliphatic content and higher charge, or regions of helical and disordered propensities. These clusters uncouple amyloid propensity from solubility representing sequence flavours compatible with surface-exposed patches in globular proteins, functional amyloids or sequences associated to liquid-liquid phase transitions.

\footnotetext{
${ }^{1}$ Switch Laboratory, VIB Center for Brain and Disease Research, Herestraat 49, 3000 Leuven, Belgium. ${ }^{2}$ Switch Laboratory, Department of Cellular and

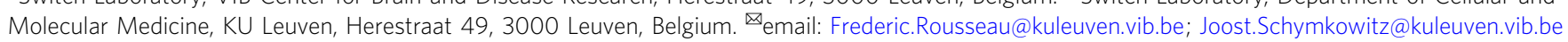


T he amyloid cross- $\beta$ state is a polypeptide conformation that is adopted by 36 proteins or peptides associated to human protein deposition pathologies ${ }^{1}$. It also constitutes the structural core of a growing number of functional amyloids in both bacteria and eukaryotes ${ }^{2,3}$. Beyond these bona fide functional and pathological amyloids it has been demonstrated that many if not most proteins can adopt an amyloid-like conformation upon unfolding/misfolding ${ }^{4}$. This has led to the notion that just like the $\alpha$-helix or $\beta$-sheet, the amyloid state is a generic polypeptide backbone conformation but also that amino acids have different propensities to adopt the amyloid conformation ${ }^{5}$. Initially, it was observed that amyloid-like aggregation correlates with hydrophobicity, $\beta$-strand propensity, and (lack of) net charge ${ }^{6}$. This triggered the development of aggregation prediction algorithms that essentially evaluate the above biophysical propensities ${ }^{7,8}$. Others extended to scaling residue propensities between protein folding and aggregation ${ }^{9,10}$. These algorithms confirmed the ubiquity of amyloid-like propensity in natural protein sequences and particularly in globular proteins as it was estimated that $15-20 \%$ of residues in a typical globular domain are within aggregation-prone regions (APRs) ${ }^{11,12}$. These APRs are sequence segments of six to seven amino acids in length on average and are mostly buried within the protein structure where they constitute the hydrophobic core stabilising tertiary protein structure $^{13-15}$. On the other hand, the increasing identification of both yeast prions and functional amyloids clearly indicated that amyloid sequence space is not monolithic and that more polar/ less aliphatic sequences represent important alternative populations of amyloid sequence space ${ }^{3}$. The limited sensitivity of the above cited algorithms to specifically identify these other subpopulations confirmed the underestimated sequence versatility of the amyloid conformation. Indeed, more recently the role of amyloid-like sequences in proteins mediating liquid-liquid phase transitions again demonstrates the ubiquity of the amyloid in biological function and further withers the image of the amyloid state as a predominantly disease and/or toxicity-associated protein conformation ${ }^{16-18}$. Rather, this suggests that like globular protein folding, amyloid assembly is a matter of kinetic and thermodynamic control that can be evolutionary tuned by sequence variation and selection.

Efforts to develop aggregation predictors that can identify a broader spectrum of amyloid sequences have increased over the years ${ }^{19}$. Such approaches focused on identifying position-specific patterns by reference to accumulated experimental data of $\mathrm{APRs}^{20-22}$, or by using energy functions of cross-beta pairings ${ }^{23}$. Recently developed meta-predictors produce consensus outputs by combining previous methods, in an attempt to boost performance $e^{24,25}$. Indirect structure-based methods were initially developed by considering secondary structure propensities ${ }^{26,27}$. Complementary studies extended this notion by suggesting that disease-related amyloids form $\beta$-strand-loop- $\beta$-strand motifs ${ }^{28}$. However, the principle of using structural information to accurately predict aggregation prone segments in protein sequences stems from the detailed work of Eisenberg and co-workers. The 3D-profiling method utilised the crystal structure of the fibrilforming segment NNQQNY (PDB ID: 1YJO) derived from the Sup35 prion protein, to thread and evaluate sequence fitting using the Rosetta energy function ${ }^{29}$. In this work, we build on this principle to develop Cordax, an exhaustively trained regression model that leverages a substantial library of curated template structures combined with machine learning. Cordax not only detects APRs in proteins, but also predicts the structural topology, orientation and overall architecture of the resulting putative fibril core. To validate the accuracy of our predictions, we designed a screen of 96 newly predicted APRs and experimentally determined their aggregation properties. Using this approach, we identified less hydrophobic polar and charged aggregation prone sequences that increasingly uncouple solubility and amyloid propensity, closely resembling characteristics of phase-separation inducers. Clustering by $\mathrm{t}$-distributed stochastic neighbour embedding reveals the heterogeneous substructure of amyloid sequence space consisting in varying clusters corresponding to sequences compatible with globular structure, functional scaffolding amyloids, N/Q/Y-rich prions, helical peptides and intrinsically disordered sequences. Together, the structural exploration performed here demonstrates that the field now gathered sufficient structural and sequence information to start classifying amyloids according to different structural and functional niches. Just like for globular proteins in the 1980s, this will allow to fine-tune both general and context-dependent structural rule learning allowing to manipulate and design amyloid structure and function.

\section{Results}

Overall approach of Cordax. We wanted to design a novel structure-based amyloid core sequence prediction method that (a) leverages all the available structure information that is currently available, and (b) employs a machine-learning element for optimal prediction performance. To this end, we first built a curated template library of amyloid core structures as described in the paragraph below. In the vein of previous prediction methods ${ }^{29}$, we fixed on the hexapeptide as a unit of prediction. In order to determine the amyloid propensity of a query hexapeptide we start by modelling its side chains on all the available amyloid template structures using the FoldX force field ${ }^{30}$, which yields a model and an associated free energy estimate $(\Delta G, \mathrm{kcal} / \mathrm{mol})$ for each template. These free energies are then fed into a logistic regression model, which is a simple statistical method relating a binary outcome to continuous variables. The prediction output of Cordax is multiple: First, there is the prediction from the logistic regression whether or not the segment is an amyloid core sequence. Second, for the sequences deemed amyloid core, the most likely amyloid core model is provided. For longer query sequences, a sliding window approach is adopted. The technical details of the pipeline can be found in the "Methods" section.

Refinement of fibril structures for machine learning. We isolated 78 short segment fibril core high-resolution structures from the Protein Data Bank (Supplementary Data 1). Templates were grouped into seven distinct topological classes out of eight theoretically possible based on their overall structural properties, as previously proposed by Sawaya et al. ${ }^{31}$. Briefly, topologies are defined by whether $\beta$-sheets have parallel versus antiparallel orientation, by the orientation of the strand faces that form the steric zipper (face-to-face versus face-to-back), and finally the orientation of both sheets towards each other and whether that results in identical or different fibril edges. This complexity was addressed by generating an ensemble of amyloid cores per structure using crystal contact information derived from the solved structures. Every template comprises two facing $\beta$-sheets, each composed of five successive $\beta$-strands. Since parallel architectures can share more than one homotypic packing interface, those structures were split into separate individual entries (Fig. 1). To ensure uniformity, we expanded the number of structural variants by breaking down longer segments into hexapeptide constituents, thus yielding a library of 179 peptide fragment structures (Fig. 1 and Supplementary Data 1).

The amyloid interaction interfaces were analysed in detail following energy refinement by the FoldX force field ${ }^{30}$. During this step we identified and rejected 33 imperfect $\beta$-packing interfaces formed by $\beta$-strands that contribute less than three 


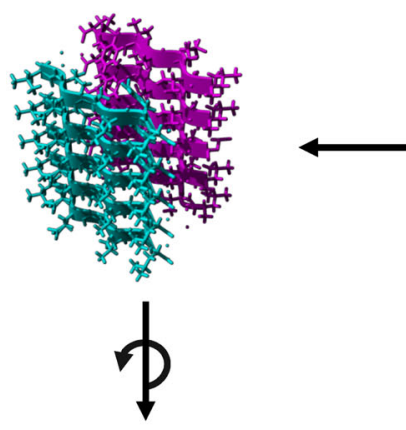

$90^{\circ}$

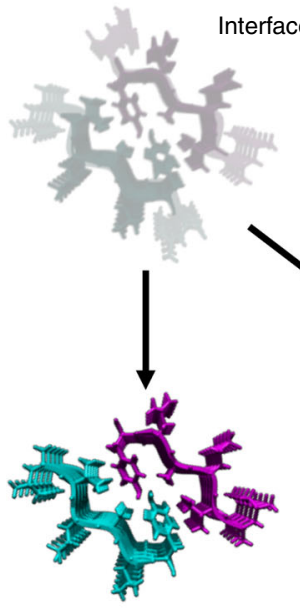

Fragment a_1

terface a

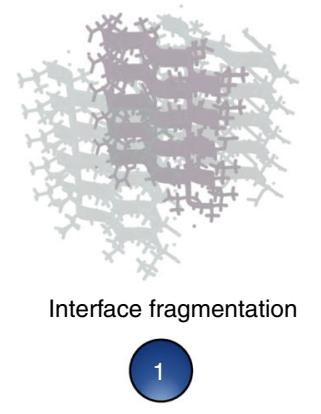

cordas Library
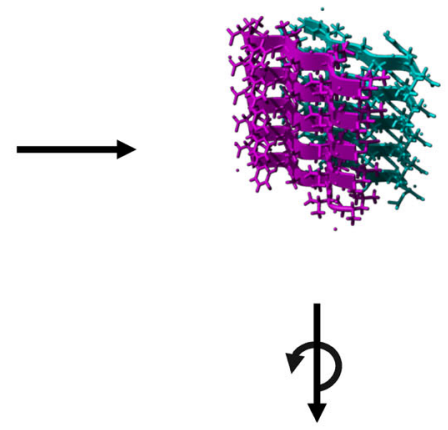

$90^{\circ}$

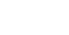

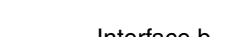

Interface b

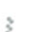


a

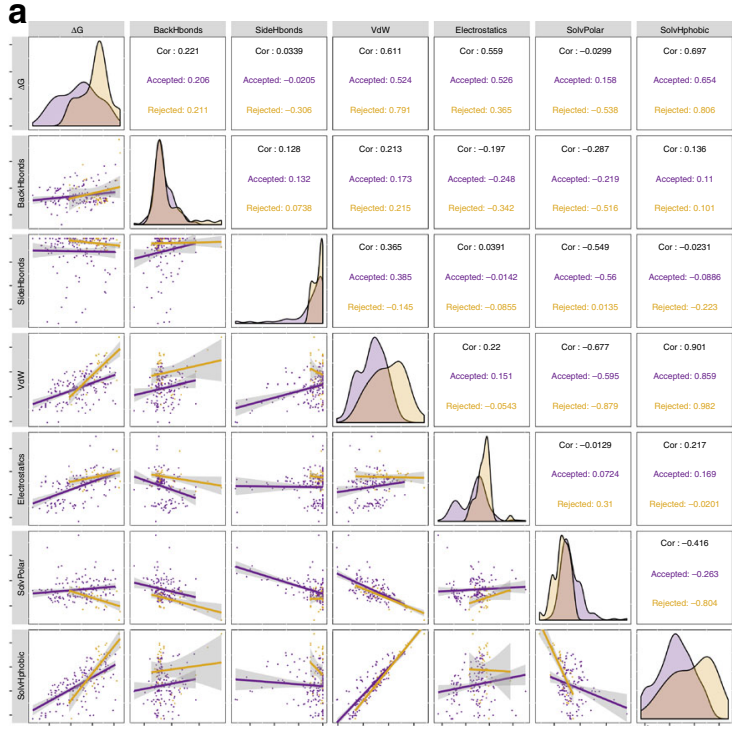

b
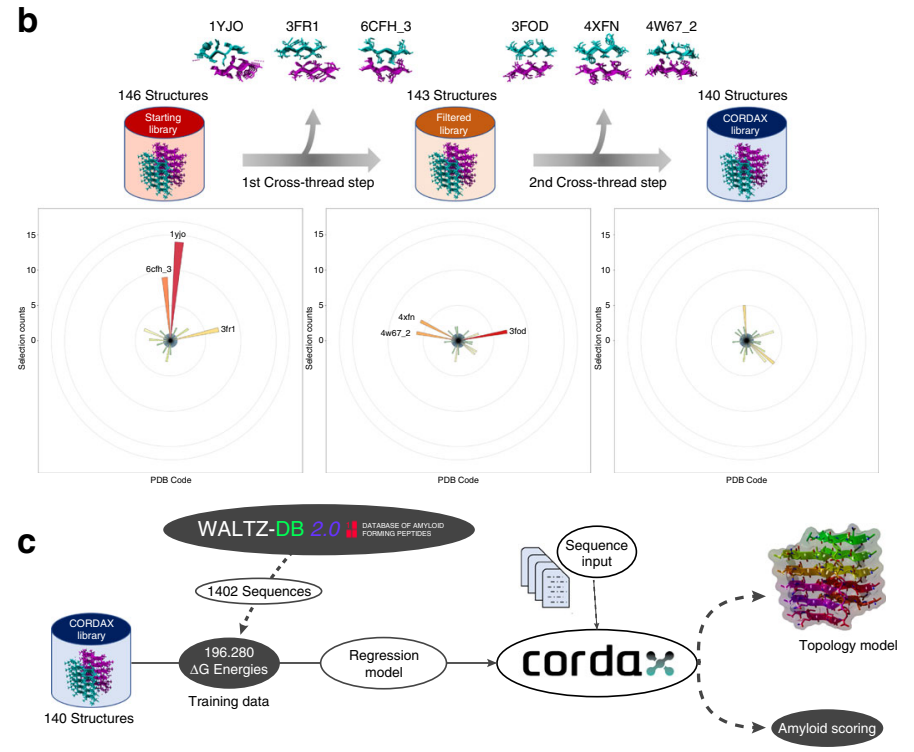

Fig. 2 Optimising the Cordax structural library. a Correlation plot of interface energies calculated using FoldX. Top half shows correlation values with scatter plots indicated at the bottom half. Rejected fragments sharing low shape complementarity (shown in yellow) have correlating weak van der Waals interfaces, as well as poor solvation energies for hydrophobic side chains compared to the remaining library (indicated in purple). Linear regression lines are shown with 95\% confidence interval (shown in grey shaded areas). b Promiscuity sorting of the structural library performed as a two-step crossthreading process. Circular histograms highlight three major promiscuous structures $(n>5)$ which were removed during the primary (PDB ID: 1YJO, 3FR1 and 6CFH_3) and secondary step (PDB ID: 3FOD, 4XFN and 4W67_2). c Schematic representation of Cordax training and the derived pipeline.

a

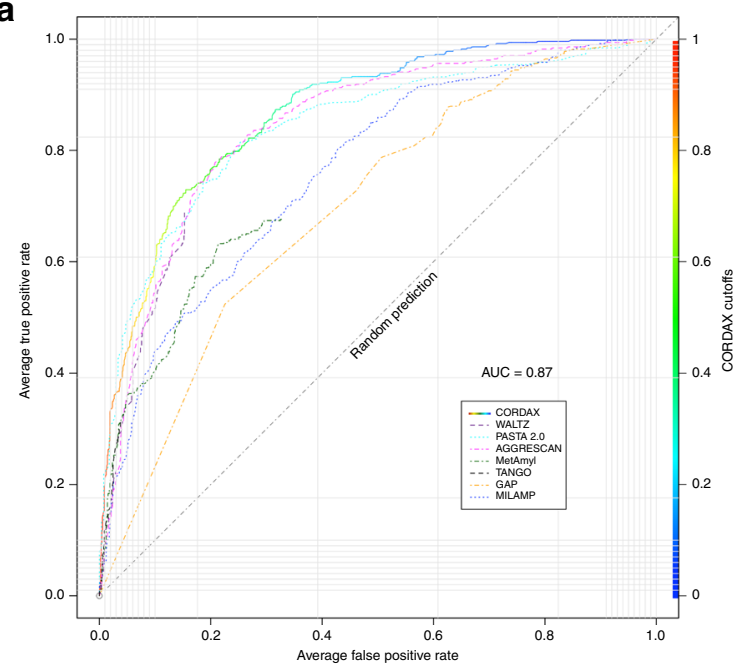

C

\begin{tabular}{cc|c|c|c|c|c|c|c} 
C & CORDAX & WALTZ & PASTA 2.0 & AGGRESCAN & MeAAmyl & TANGO & GAP & MILAMP \\
Accuracy & 0.81 & 0.67 & 0.76 & 0.57 & 0.79 & 0.73 & 0.51 & 0.73 \\
\hline Precision & 0.74 & 0.54 & 0.74 & 0.60 & 0.72 & 0.82 & 0.42 & 0.66 \\
\hline TPR (Recall) & 0.72 & 0.65 & 0.52 & 0.85 & 0.69 & 0.31 & 0.94 & 0.49 \\
\hline FPR & 0.14 & 0.33 & 0.10 & 0.54 & 0.15 & 0.04 & 0.74 & 0.14 \\
\hline MCC & 0.57 & 0.34 & 0.46 & 0.29 & 0.54 & 0.38 & 0.25 & 0.38 \\
\hline F1 & 0.73 & 0.60 & 0.61 & 0.71 & 0.70 & 0.45 & 0.58 & 0.56 \\
\hline AUC & 0.87 & 0.73 & 0.84 & 0.84 & 0.78 & 0.64 & 0.70 & 0.76
\end{tabular}

b
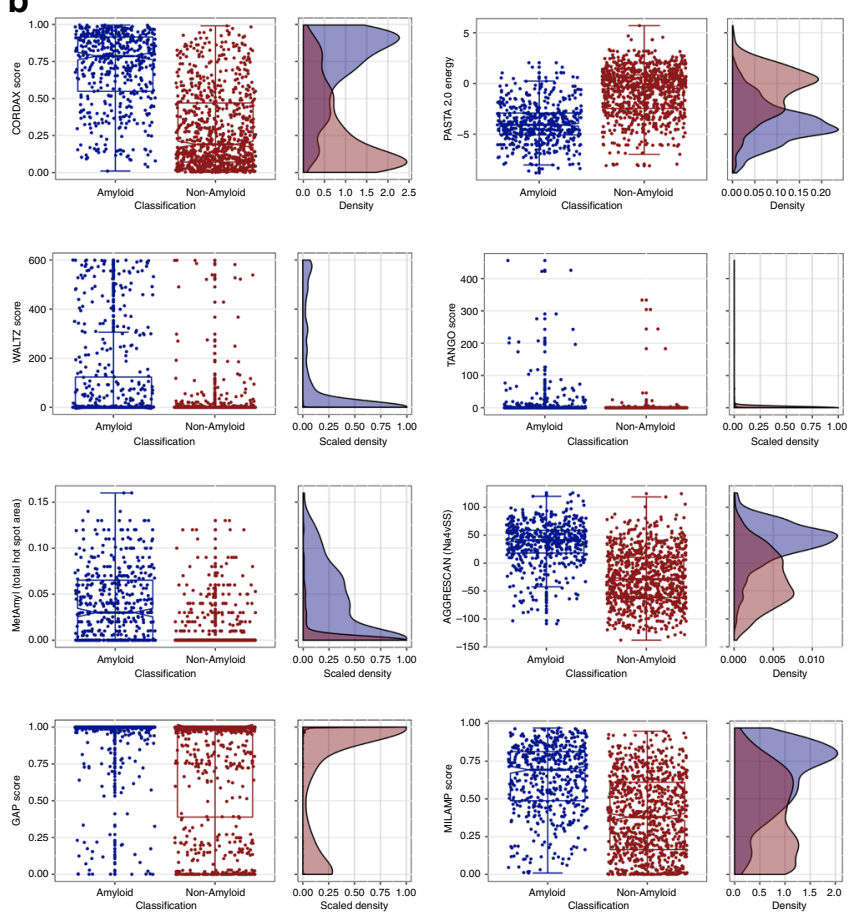

Fig. 3 Benchmarking of CORDAX. a ROC curve analysis for Cordax and seven other state-of-art methods against WALTZ-DB 2.0. For WALTZ, TANGO and MetAmyl, FPR stops at earlier rates due to minimal scoring variations. b Cordax score distribution compared to other tools. The regression model achieves better scoring separation for predictions between amyloid-forming (shown in blue) and non-amyloid sequences (shown in red). Distributions are shown as standard box-plots highlighting median, upper (Q3) and lower (Q1) quartiles. Whiskers indicate the ranges for values within 1.5 interquartile range (IQR) above and below Q3 and Q1, respectively. Density plots for WALTZ, TANGO, MetAmyl and GAP are scaled due to the overrepresentation of unscored values or false positives, respectively. $\mathbf{c}$ Performance metrics comparison indicating Cordax superiority to other sequence predictors $(\mathrm{MCC}=$ $0.57, \mathrm{~F} 1=0.73$ and $\mathrm{AUC}=0.87$ ). 
the training data set. Despite its wide use, this collection suffers from insufficient experimental characterisation of certain large entries (i.e. gelsolin, kerato-epithelin, lactoferrin, amphoterin and others), which has been shown to introduce type-I errors (false positives). This error propensity derives from non-amyloid annotations which primarily correspond to regions of undetermined aggregation propensity, a notion that is highlighted by recent studies, such as in the case of calcitonin ${ }^{35}$, cystatin- $\mathrm{C}^{36}$ and transthyretin ${ }^{37}$. In contrast, other proteins have been linked to the formation of $\beta$-helical structures and as an after effect contain elongated fragments characterised, yet unverified in their entirety, as amyloidogenic, which can introduce type-II errors (false negatives) when applying predictors of local aggregation propensity ${ }^{38-41}$. The aforementioned shortcomings are reflected by the low MCC values that are reported for all aggregation predictors (Supplementary Table 1) and the fact that predicted segments were originally considered neutral, but later shown to be aggregation hotspots (Supplementary Fig. 1) 15-41. $^{35}$

Designed APR nucleators validate the accuracy of Cordax predictions. In the interest of improving the current description of the familiar amyloidogenic protein dataset, we selected and synthesised a subset of 96 peptides corresponding to strong aggregation prone regions identified in these proteins by Cordax. Apart of prediction strength, the peptide screen was also selectively constructed to ensure broad sequence variability and a wide distribution on the proteins of the dataset, with a preference for longer entries defined by inadequate previous characterisation. Peptide sequences were cross-checked and filtered to exclude overlapping sequences with previously identified amyloid regions and WALTZ-DB (Supplementary Data 2). The remaining selection of 96 peptides were synthesised using standard solid phase synthesis and their amyloid-forming properties were initially examined using Thioflavin-T (Th-T) or pFTAA binding, following rotating incubation for 5 days at room temperature. The binding assays are complementary, as Th- T and pFTAA are opposingly charged molecules, which increases the amyloid identification rate by overcoming cases of dye-specific failure to bind to amyloid surfaces based on charge repulsion. Under these conditions, 66 peptides successfully bind to the specific dyes (Fig. 4a and b) by forming fibrils with typical amyloid morphologies and properties that were verified using transmission electron microscopy (Fig. 4c) and Congo red staining for selected cases (Fig. 4d). As these dyes are known to yield false negatives, in particular for short peptides, all dye-negative peptides were further investigated using electron microscopy. During this scan, we recovered 19 additional sequences that were capable of forming sparse amyloid-like fibrils with shorter lengths (Supplementary Fig. 2). Taking the latter into account, Cordax was able to fish out a total number of 85 novel nucleation segments with unparalleled accuracy (89\%), thus providing a rigorously improved description of the protein set to be used for the efficient testing and development of future predictors (Supplementary Fig. 1).

Cordax detects highly soluble surface-exposed conformational switches. The expanded amyloidogenic annotation of the protein dataset was supplemented with structural analysis of the newly identified aggregation prone regions. Out of 96 peptides designed and experimentally tested, 85 peptides were found to display evident amyloid-forming features, with more than half (55.3\%) being predicted specifically by Cordax, contrary to shared predictions with sequence-based tools of high specificity (44.7\%) (Supplementary Data 2). Pinpointing the location of the identified nucleators in parental protein folds (Fig. 5a) revealed that APRs picked up both by Cordax and traditional sequence-based methods are usually found buried within the core of soluble proteins. Contrary to what has been previously reported ${ }^{14,15}$, however, our regression model also discovered additional nucleating sequences that primarily appear to reside on the surface of protein molecules (Fig. 5b-h) and as a result, are characterised by high solvent exposure (Fig. 5i and j). Partition coefficients clearly indicate that these exposed peptide segments identified by Cordax are primarily water-soluble sequences, whereas APRs that are predicted by the majority of sequence-based predictors are largely insoluble (Fig. 5k). Sequence distribution analysis signifies that this increased exposure and solubility is complemented by an expected decrease in sequence hydrophobicity (Fig. 5l). More specifically, APRs identified solely by Cordax are relatively enriched in charged or polar side chains (Fig. 5l) and are frequently parts of a-helical or unstructured segments (Fig. $5 \mathrm{~m}$ ). This implies that these regions are in fact conformational switches that may, under fitting misfolding conditions, transiently move towards the formation of $\beta$-aggregates. The fact that these sequences are not dictated by typical sequence propensities, such as hydrophobicity or $\beta$-structure tendency, explains why sequence-based predictors overlook them.

Cordax infiltrates uncharted areas of amyloid sequence space. To further explore the capabilities of our method, we composed a map of the known amyloid-forming sequence space using $\mathrm{t}$ distributed stochastic neighbour embedding (t-SNE) for dimensionality reduction (Fig. 6a). As input, we used a 20-dimensional parameterisation vector describing all newly identified amyloidogenic peptides merged to the known amyloid-forming hexapeptide sequences in WALTZ-DB, in terms of their basic physicochemical properties and amino acid composition, as well as prediction outputs derived from Cordax and other high specificity predictors. t-SNE mapping pinpointed clear areas of sequence space where Cordax correctly identifies amyloid propensity (purple colour in Fig. 6a), which primarily extend towards regions that remain unpredicted (shown in black) and seclude from a large base of sequences identified by multiple methods, including Cordax (cyan colour). Clustering analysis (Fig. 6b) performed using physicochemical properties (Figs. 6c-e), secondary structure propensities (Fig. 6f) and side chain size distributions (Fig. 6g, h) identifies that this common base of by-now easy to predict APRs are characterised by high hydrophobicity, strong $\beta$-sheet propensity and a high relative content of aliphatic side chains (cluster 1 in Fig. 6b), still echoing the initial discovery of APRs by these features ${ }^{6}$. Cordax explores regions adjacent to this with a higher content of shorter side chains (clusters 2 and 5). Notably, amyloid nucleators of this composition are an invaluable resource for amyloid nanomaterial designs with elastin-like properties, are enriched in functional amyloids and have also been linked to ancestral amyloid scaffolds in early life ${ }^{42-45}$. A similar trend in amino acid composition has also been reported for proteins that form condensates through phase transition, such as TDP-43 and FUS ${ }^{16,18}$. Low complexity regions (LCRs) that are enriched in short side chains, such as Gly or Ala, have been shown to drive phase separation, often as an intermediate event towards fibrillation, particularly in polar LCRs with lower aliphatic content and strong disorder or $\alpha$-helical propensities, such as the sequences discovered in cluster $5^{17,46}$. Further to this, Cordax provides significant advancement by traversing in areas with a higher content of negatively or positively charged regions (clusters 3, 4, 6 and 7, respectively). Charged residues often act as gatekeepers that directly disrupt aggregation or modulate it by flanking APRs within protein sequences ${ }^{4}$. Based on this premise, most sequence-based predictors negatively correlate net charge to 

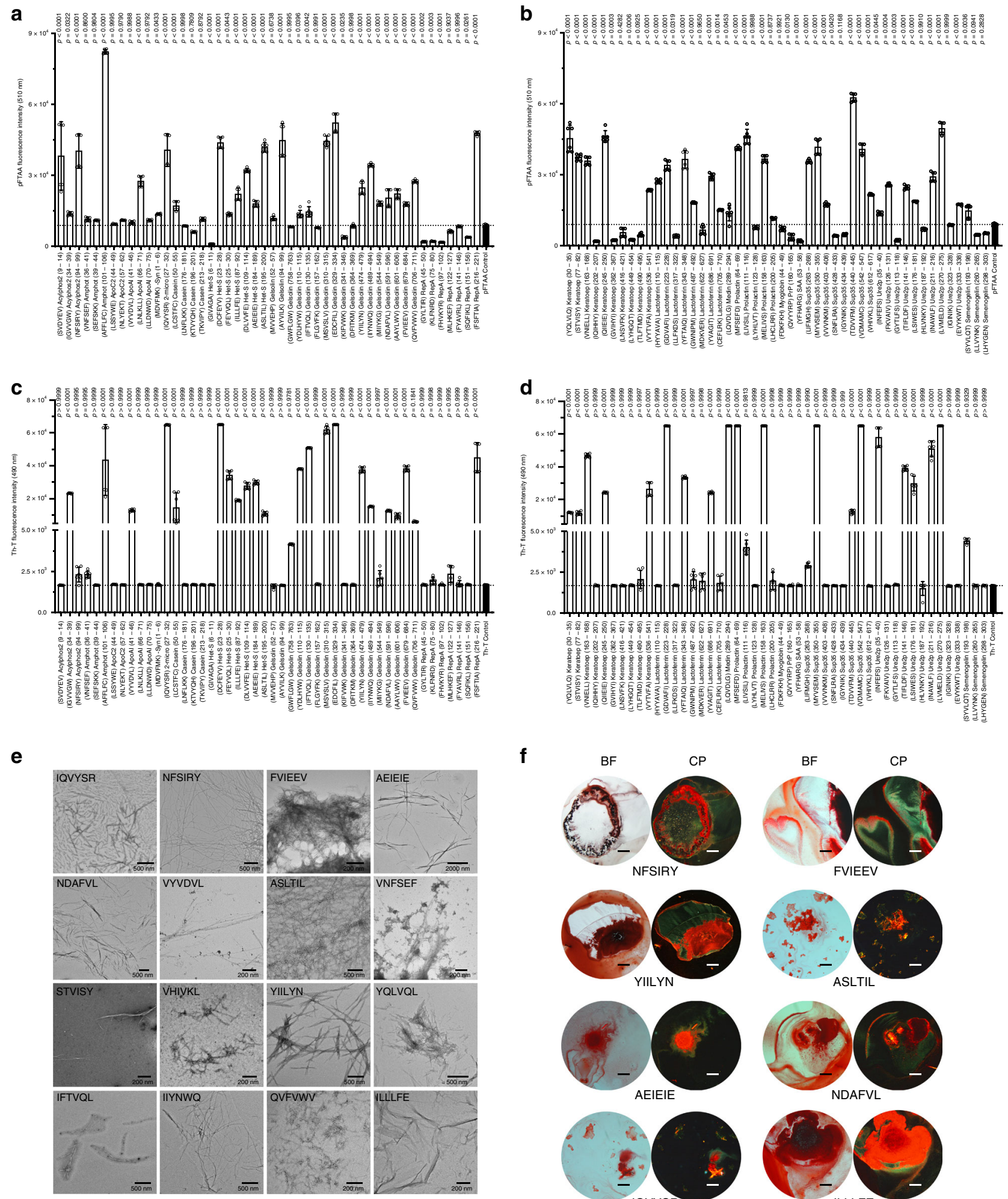

$\mathbf{f}$
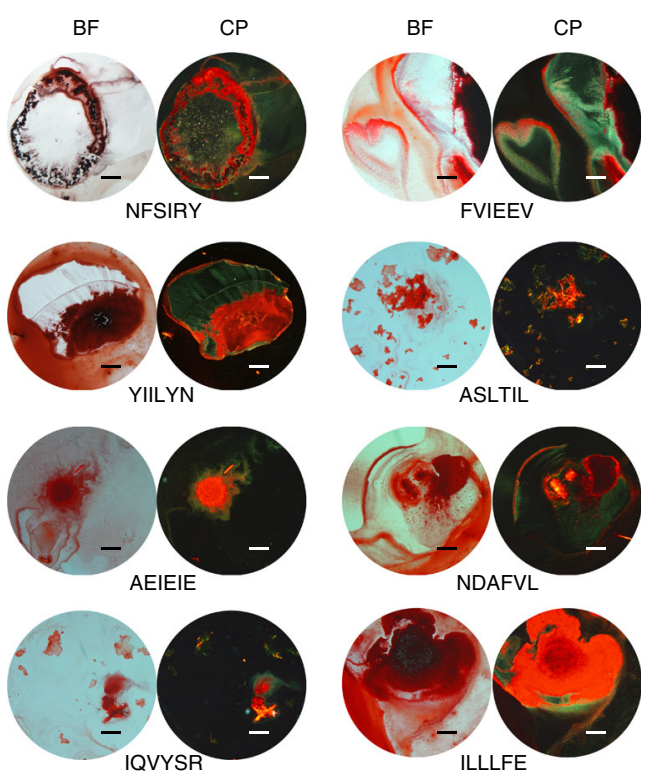

Fig. 4 Amyloid-forming properties of the peptide screen designed by employing Cordax. $\mathbf{a}$ and $\mathbf{b}$ Measured pFTAA and $\mathbf{c}$ and $\mathbf{d}$ Th-T fluorescence of synthetic peptides following rotation at $200 \mu \mathrm{M}$ for 5 days. Data represents mean \pm SD $(n=6$ independent experiments, statistics: one-way ANOVA with multiple comparison against the vehicle control). e Electron micrographs of amyloid fibrils formed by Th-T or pFTAA-binding peptides. $\mathbf{f}$ Suspensions of amyloid fibrils bind Congo red as displayed under bright field illumination (BF) and exhibit typical for amyloids apple-green birefringence under crossedpolarised light (CP). Scale bars: $500 \mu \mathrm{m}$. Source data are provided as a Source Data file. 
a

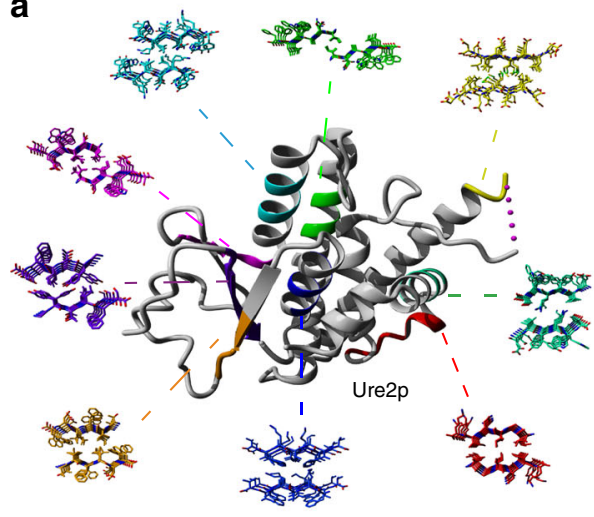

b

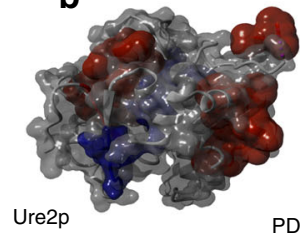

PDB: $1 \mathrm{HQO}$

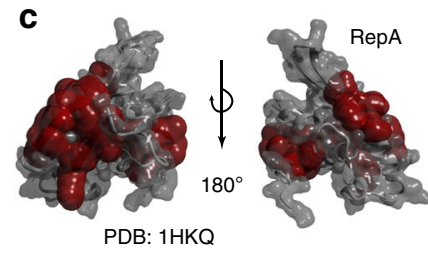

C

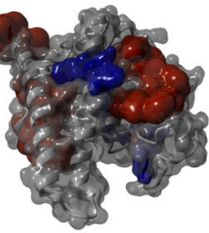

d

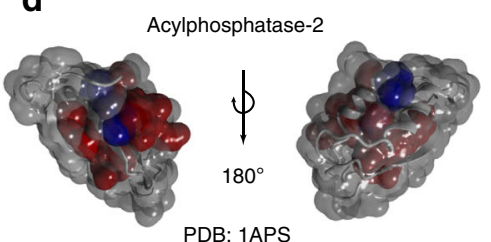

PDB: 4CRN
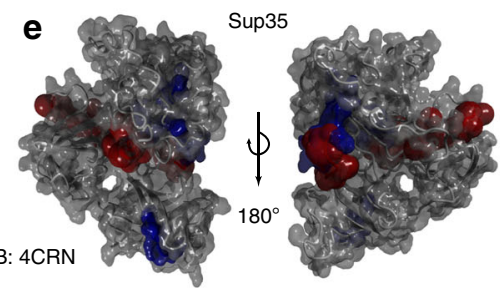
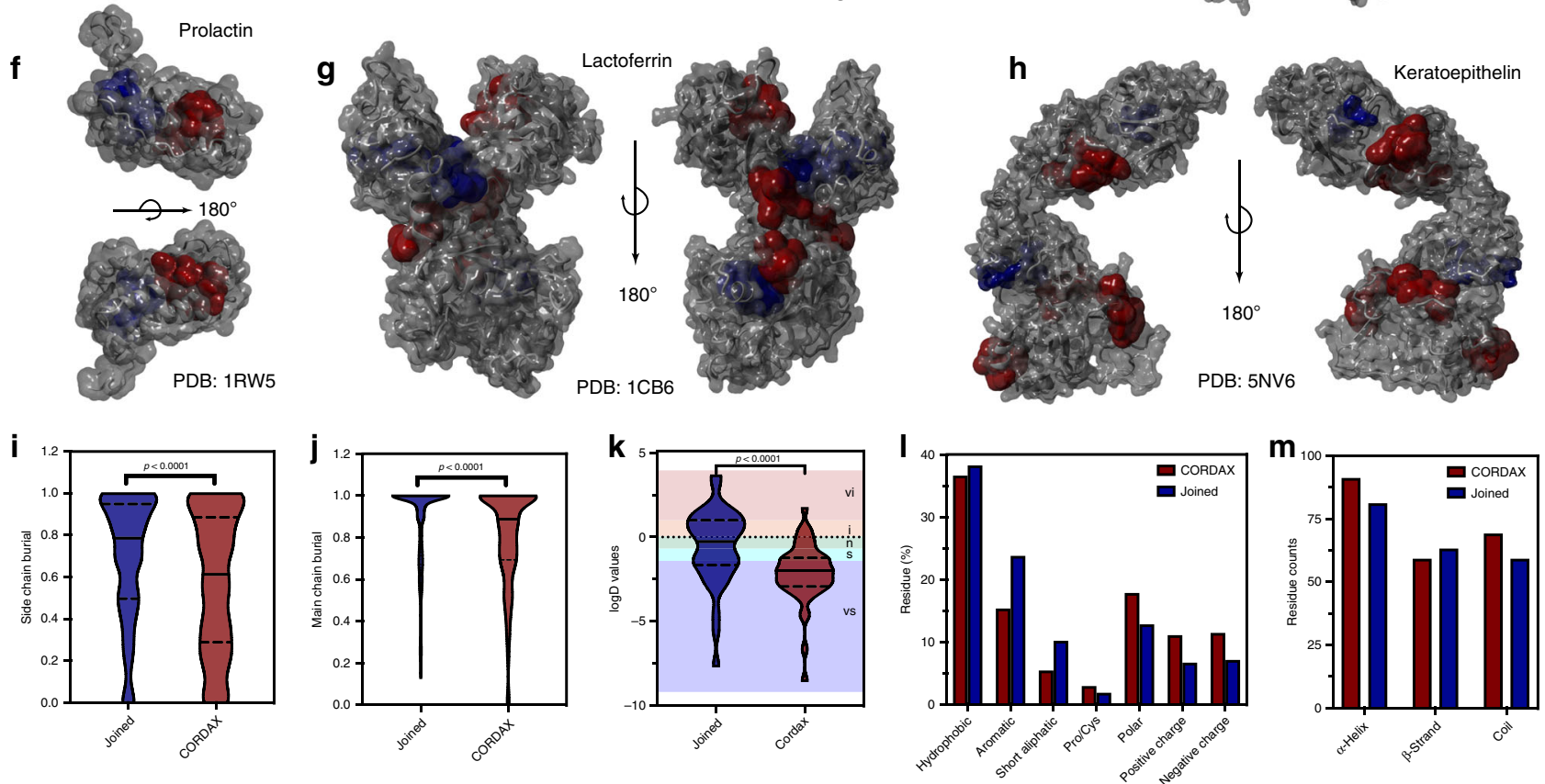

Fig. 5 Cordax identifies surface-exposed aggregation nucleators spanning residues that are typically considered unconventional for amyloid fibril formation. a Schematic representation of Cordax-predicted topological models for APRs charted against the cognate native crystal structure of the amyloidogenic protein Ure2p. b-h Surface representation of folded structures for b Ure2p, c RepA, d Acylphosphatase-2, e Sup35, f Prolactin, g Lactoferrin and $\mathbf{h}$ Kerato-epithelin reveals that aggregation nucleators uniquely identified by Cordax (highlighted in red) are primarily exposed to the surface of proteins, compared to segments of joint prediction (shown in blue) which are predominantly buried within the hydrophobic core of the native fold. Cordaxspecific predicted APRs produced lower volumetric burial values, calculated using FoldX, for $\mathbf{i}$ side chain and $\mathbf{j}$ main chain groups indicating that they are considerably exposed compared to jointly identified nucleators. $\mathbf{k}$ Partition coefficients indicate that Cordax-specific APRs are significantly more soluble compared to typically predicted sequences that are primarily hydrophobic and therefore insoluble. Solubility regions (vi very insoluble, i insoluble, $\mathrm{n}$ neutral, s soluble, vs very soluble) are shown as coloured backgrounds ${ }^{72}$. Significant differences were computed using one-way ANOVA with multiple comparison. I Surface-exposed Cordax-specific APRs are composed of residues with a $20 \%$ increase in polar and charged side chains, in expense of hydrophobic residues ( $n=219$ residues in APRs identified by Cordax, $n=203$ residues in APRs from joined predictions). $\mathbf{m}$ Secondary structure analysis, using FoldX, indicates that Cordax identifies several APRs that reside in $\alpha$-helical or unstructured regions within the native fold, suggesting that amyloidogenic proteins may harbour a plethora of exposed conformation switches that can act as potential nucleators of amyloid fibril formation, under suitable misfolding conditions ( $n=219$ residues in APRs identified by Cordax, $n=203$ residues in APRs from joined predictions). Violin plots represent the kernel probability densities of the data with the median, upper and bottom quartiles. Source data are provided as a Source Data file.

protein aggregation and have increased failure rates when identifying such amyloid-forming stretches. On the other hand, sequences with a high content of aromatic side chains are relatively easy to identify (clusters $9 a$ and $9 b$ ), following several lines of evidence supporting their role in amyloid fibril formation ${ }^{48}$. Cordax also pushes forward into less well-charted areas of amyloid sequence space, e.g. exploring clusters with high $\alpha$-helical content (cluster 10) and overall a low content of aliphatic amino acids (clusters 5, 6, 7, 8 and 9b). These regions also reveal the scope to improve the method, as in particular, the region with high disorder propensity (cluster 11) still contains many false negatives, in spite of the ability of Cordax to partially pick up a minority of sequences. Interestingly, a closer look at the partition coefficients of the known amyloid sequence space reveals that although Cordax takes a significant step forward towards the right direction, these APRs remain very hard to identify as they are characterised by even higher solubility values (Fig. 6i). Similar charting of the amyloid sequence space is achieved by using 

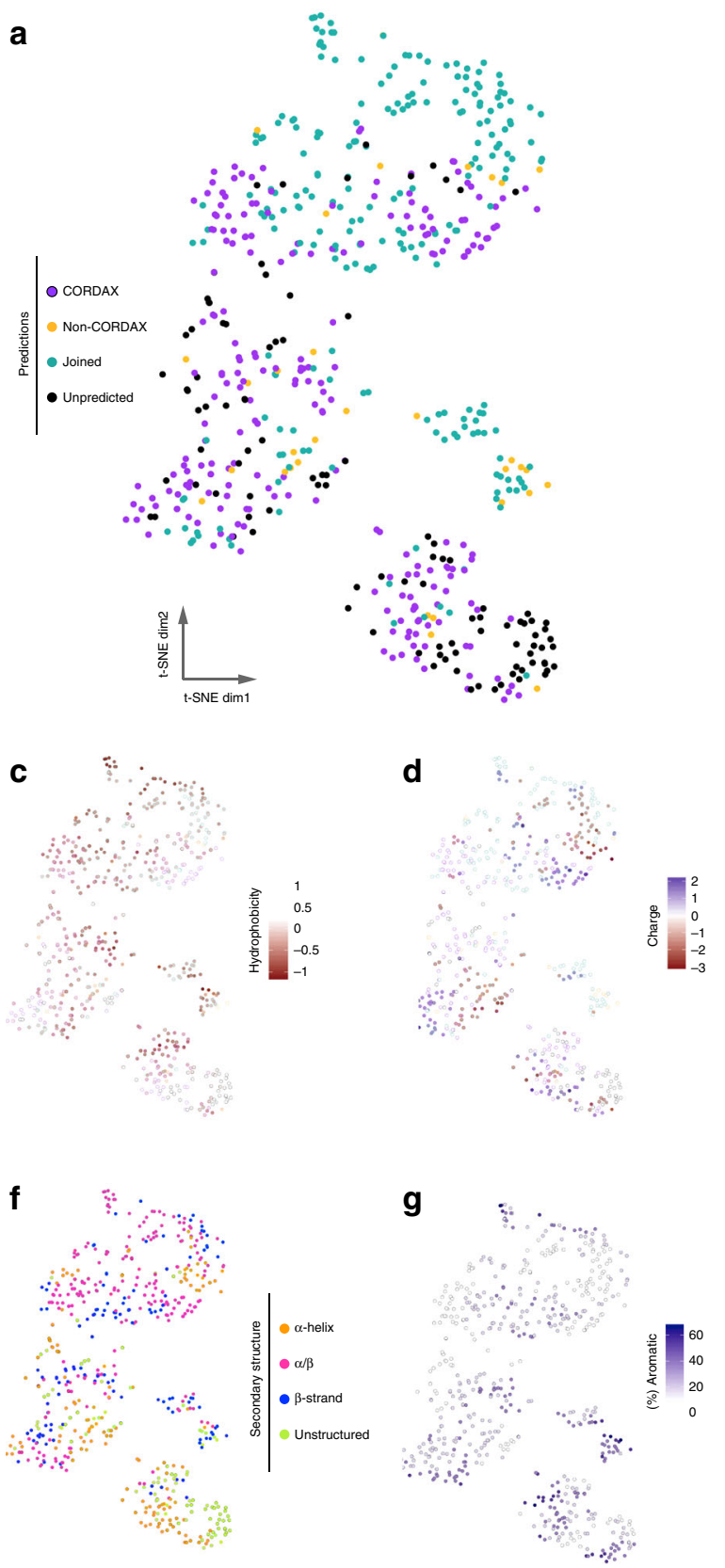
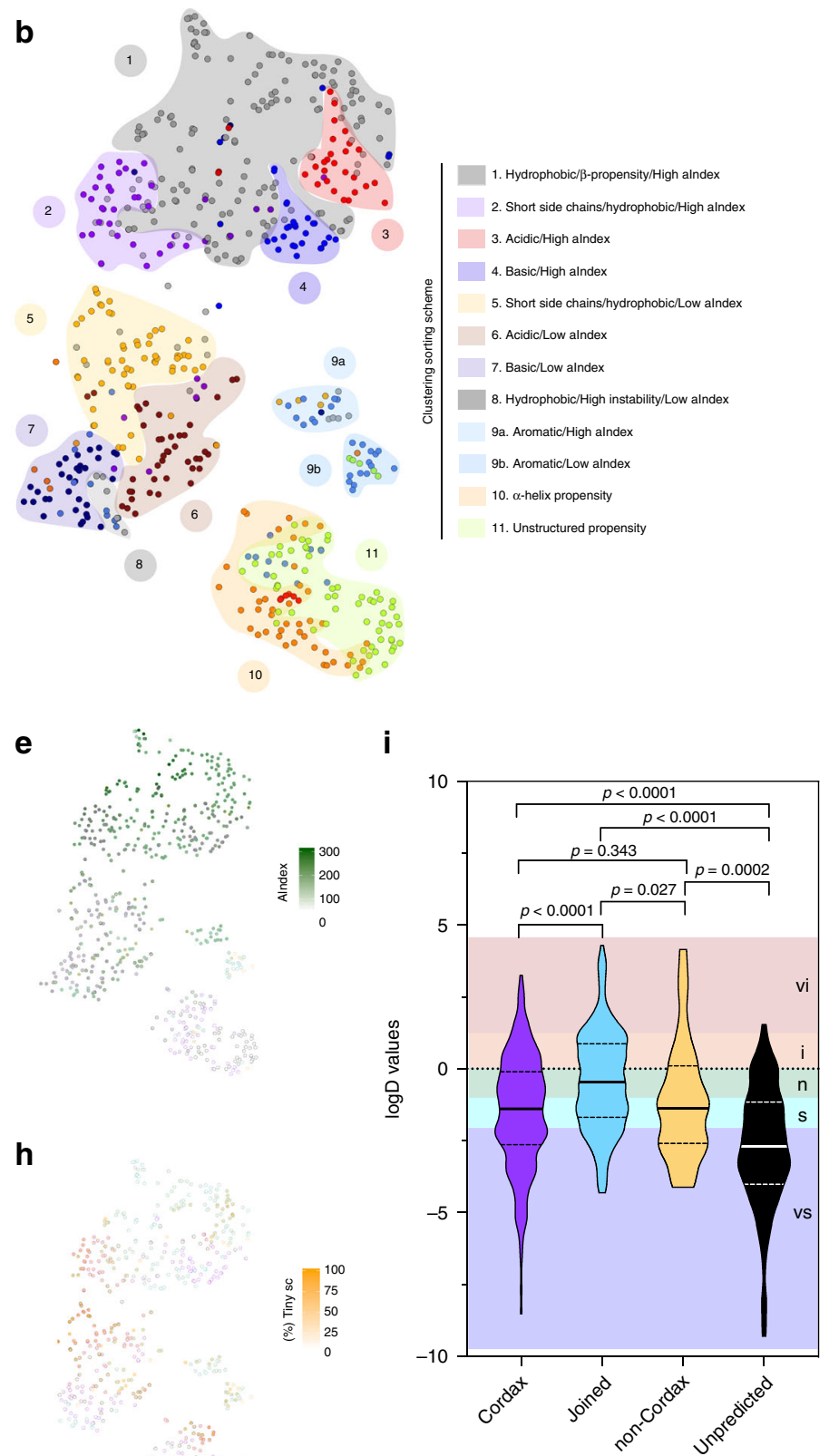

Fig. 6 t-SNE 2D-representation of the known experimentally determined amyloidogenic sequence space. a State-of-the-art sequence-based methods predict amyloid sequences, with (shown in cyan) or without Cordax (shown in yellow), that are grouped together in a major landing cluster and two islands. Cordax predictions (shown in purple) transgress towards areas of amyloid-forming sequences that remain undetected by most methods (shown in black). $\mathbf{b}$ Clustering of the t-SNE map using basic physicochemical properties and amino acid composition of the amyloid peptides. Each data point is colour-coded based on the sorting scheme shown in the legend and background areas are used to pinpoint the major clusters of each defined category. The clustering scheme was defined by characterising the t-SNE map using peptide $\mathbf{c}$ hydrophobicity, $\mathbf{d}$ net charge, e aliphatic index, $\mathbf{f}$ secondary structure propensity and percentage content of $\mathbf{g}$ aromatic or $\mathbf{h}$ short residue side chains. $\mathbf{i}$ Partition coefficient analysis reveals that APRs identified by Cordax are primarily soluble sequences compared to easy to identify sequences of joint prediction. On the other hand, APRs that remain hard to detect are characterised by higher solubilities. Solubility regions (vi very insoluble, i insoluble, $\mathrm{n}$ neutral, s soluble, vs very soluble) are shown as coloured backgrounds. Significant differences were computed using one-way ANOVA with multiple comparison. Violin plots represent the kernel probability densities of the data with the median, upper and bottom quartiles.

uniform manifold approximation and projection (UMAP) for dimensionality reduction (Supplementary Fig. 3a and b), while PCA analysis highlights that CORDAX slowly infiltrates the sequence space of higher solubilities (Supplementary Fig. $3 \mathrm{c}$ and d). Overall, dimensionality reduction transformation highlights that structural compatibility can overcome typical sequence propensities as a pivotal driver of aggregation nucleating sequences and suggests that under the proper conditions, the boundaries currently considered compatible to protein amyloid-like assembly are potentially far wider than previously expected. 


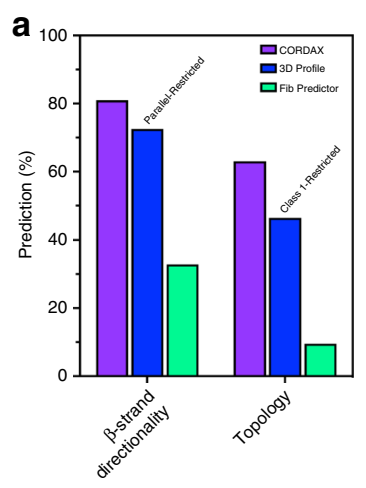

b
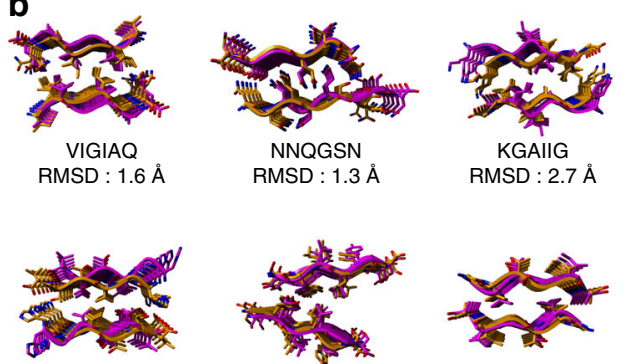

HGVTTV RMSD : $2.3 \AA$
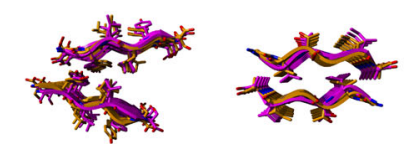

GYLLGS
RMSD : $1.1 \AA$
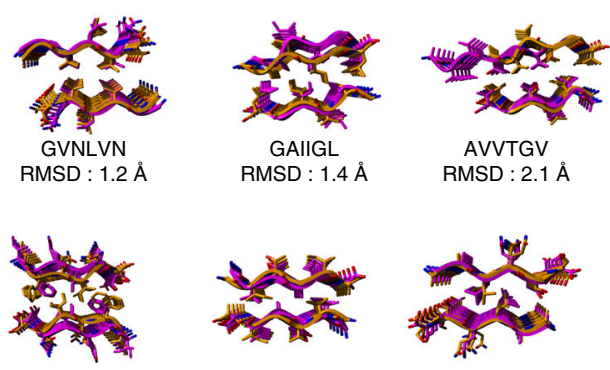

GVTGIA

RMSD : $0.9 \AA$

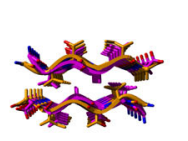

GVTAVA RMSD : $1.3 \AA$

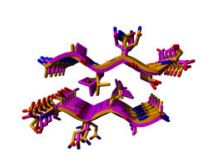

AALQSS RMSD : $1.2 \AA$

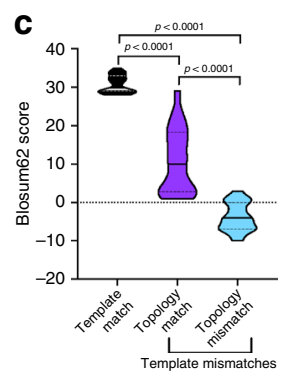

d

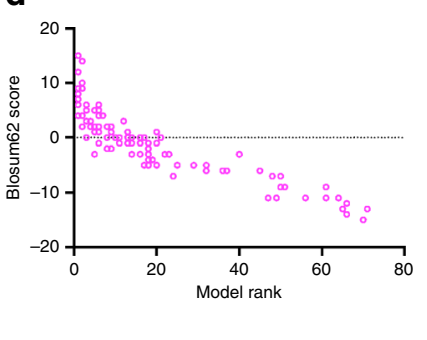

e (IYQYGG) 6G8C
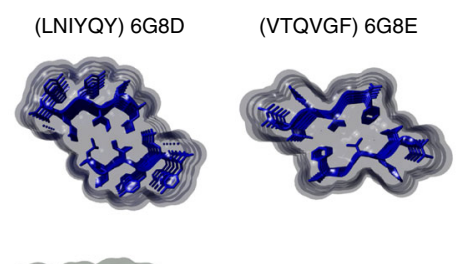

(IFAEDV) $5 \mathrm{TXH}$
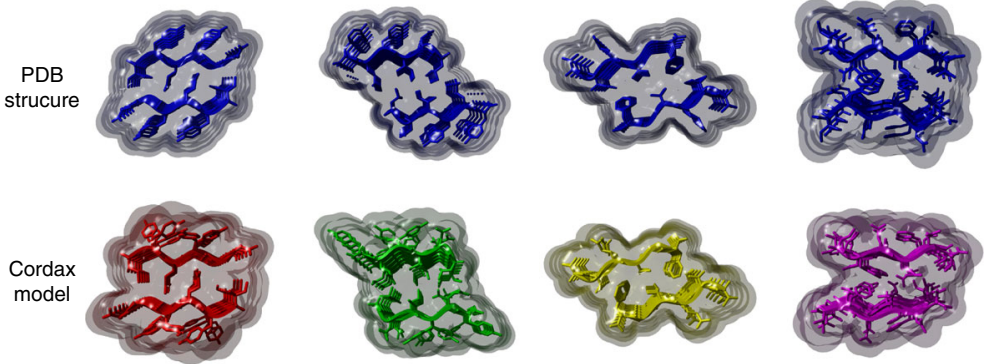

f

\begin{tabular}{|c|c|c|c|}
\hline PDB ID & CORDAX & 3D Profile & FibPredictor \\
\hline $6 \mathrm{G} 8 \mathrm{C}$ & 0.4 & 2.7 & 3.8 \\
\hline $6 \mathrm{G} 8 \mathrm{D}$ & 2.1 & 2.1 & 2.4 \\
\hline $6 \mathrm{G} 8 \mathrm{E}$ & 1.1 & 1.5 & Class mismatch \\
\hline $5 \mathrm{TXH}$ & 1.7 & Class mismatc & Class mismatch \\
\hline
\end{tabular}

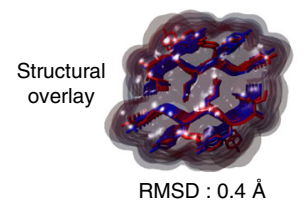

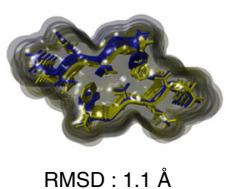

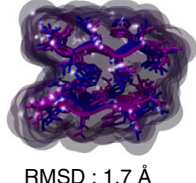

Fig. 7 High-precision recognition of amyloid fibril structural architectures using Cordax. a Prediction accuracy comparison of Cordax to the only publicly available structural predictors, Fibpredictor and 3D-profile. For comparison, methods were run against a non-redundant sequence set extracted from amyloid-forming peptide interfaces ( $n=73$ templates). b Model topologies, predicted by applying Cordax (shown in orange), strongly superimpose to matching solved structural layouts of amyloidogenic nucleators (shown in magenta), as indicated by the reported minor RMSD values. c Sequence identity contribution for template selection during cross-threading analysis of the Cordax structural library. Alignment scores for selected models matching the template sequences compared to mismatching template selections of similar or different topological layouts $(n=73$ templates). d Alignment scores of the APRs newly identified by Cordax to the sequence of the selected templates, plotted against their corresponding model ranks ( $n=96$ sequences threaded). e Structural alignment of Cordax outputs to experimentally determined 3D structures. Models were calculated for three aggregation prone sequences derived from CsgA curli forming protein (PDB IDs: 6G8C, 6G8D and 6G8E, respectively) and a peptide mutant sequence derived from A $\beta$ amyloid peptide (PDB ID: 5TXH). Predicted topologies are overlapping representations of the experimentally determined amyloid fibril cores, $\mathbf{f}$ as displayed by a direct comparison to other software. Violin plots represent the kernel probability densities of the data with the median, upper and bottom quartiles. Source data are provided as a Source Data file.

Cordax predicts the structural layout and topology of fibril cores. Due to restricted availability of experimentally determined structures not included in the Cordax library, we first analysed the information derived from cross-threading analysis in order to test the performance of the tool in predicting the structural architecture of aggregation prone stretches. Among 73 unique sequences corresponding to the structural library, Cordax was able to accurately assign the correct architecture to $63 \%$, whereas $81 \%$ was identified with proper $\beta$-strand orientation (parallel/ antiparallel) (Fig. 7a, Supplementary Data 3 and 4). In comparison, FibPredictor ${ }^{49}$ correct topology allocation was limited to $9.5 \%$ of the sequences and assigned $\beta$-strand directionality amounted to $32.9 \%$, while introducing an evident preference towards antiparallel architectures (Fig. 7a). Similarly, the 3Dprofile method is restricted to linking all potential queries with a class 1 topology, hence was incapable of predicting alternative architectures (Fig. 7a). Structural alignment indicated that even in cases of mismatching selected templates, modelled architectures strongly superimpose to the solved structures (Fig. 7b), suggesting that Cordax identifies the correct topology with high accuracy. A closer look reveals that sequence specificity may be a modulating, yet not determining factor for this selection process. Steric perturbations can be introduced due to restrictions deriving from closely interdigitating side chains within the packed interfaces, therefore, key residue positions can be bound to the overall stability of certain structural topologies and decrease the acceptable sequence space that can accommodate energetically favourable interactions. This is highlighted by the sequence similarity observed between topological matches (Fig. 7c, Supplementary Data 4). On the other hand, topologically different model selections could also be a consequential outcome of amyloid polymorphism. The observed sequence redundancy of the Cordax library illustrates that APRs can form amyloid fibrils with distinct morphological layouts ${ }^{50-52}$, a notion that is also supported by the common morphological variability of aggregates formed at the level of full-length amyloid-forming proteins ${ }^{53,54}$. The modulating role of sequence dependency was also evident for the 96peptide screen. A ranked analysis of the output models indicated that templates with higher alignment scores were not crucial for the topology selection process, although could often correspond 
to the favourable architectures (Fig. 7d), thus highlighting that the structural predictions of Cordax are relatively unbiased in terms of the sequence space composing the structural templates.

The accuracy of the tool was also cross-referenced against experimentally determined structures of fibril cores not included in the structural library. We utilised the recently solved structures of parallel fibril-forming segments derived from the major curli protein $\mathrm{CsgA}^{55}$, as well as an anti-parallel polymorphic APR variant segment derived from the amyloid- $\beta$ peptide $^{56}$. Compared to other structural predictors, only Cordax could invariantly predict the correct architecture for every steric zipper as the closest representation of the experimentally determined reference structures (Fig. 7e and f). This performance can only improve as the fragment library expands, so we aim to update it at regular intervals, providing there is a noticeable increase in solved structures in the future.

\section{Discussion}

The number of amyloid structures in the protein databank has been steadily increasing over the last two decades. It has now achieved a number $(>80)$ that was reached for globular proteins at the beginning of the 1980s and that then triggered the first developments of template-based modelling methods including homology-based and threading (or fold recognition) in an attempt to estimate the versatility of individual folds and discover novel folds in a more directed manner. Similarly, we here developed Cordax, an exhaustively trained regression model that leverages a substantial library of curated amyloid template structures combined with machine learning. Cordax uses a logistic regression approach to translate structural compatibility and interaction energies into sequence aggregation propensity and is therefore unconstrained by defined sequence tendencies, such as hydrophobicity or secondary structure preference that direct most sequence-based predictors. As a result, we discovered unconventional amyloid-like sequences, including sequences with low aliphatic content, high net charge or sequences with low intrinsic structural propensities. Clustering amyloid sequences by $\mathrm{t}$-SNE two-dimensional reduction revealed the substructure of amyloid sequence space. Apart from a large cluster corresponding to sequences found in the hydrophobic core of globular proteins, we also found clusters corresponding to surface-exposed amyloid sequences in globular proteins, small aliphatic functional amyloids, N/Q/Y prions, strongly helical and intrinsically disordered sequences which could be compatible with liquid-liquid phase responsive sequences. Our analysis highlights the discovery of highly soluble, yet amyloid-forming, sequences and suggests that the largest portion of the remaining uncharted amyloid sequence space is hidden in this corner (Fig. 6a and i). Indeed, most archetypal hydrophobic APR sequences have low intrinsic solubility. As a result, low solubility and aggregation propensity are properties that are often wrongly used interchangeably. It is important to differentiate between the initial solubility and aggregation propensity of a peptide, as soluble monomeric sequences can often self-assemble, at later time points, into insoluble amyloid fibrils. The APRs that are newly discovered by Cordax are often highly soluble in their monomeric form, even more than the already known polar APRs from the yeast prions, as they contain many charged and polar residues, yet surprisingly can still assemble into amyloids. Overall, our approach demonstrates that the increasing structural information on amyloids now allows for more fine-graded structural rule learning of the amyloid state.

Recent developments in microcrystal electron diffraction have enabled structural determination from nanocrystals that are not typically suited for traditional X-ray diffraction and have provided significant insights on the polymorphic architectures of amyloid fibrils ${ }^{57}$. In this line, the emergence of cryo-EM has been pivotal in determining features of amyloid fibril polymorphs ${ }^{58}$, complementing earlier efforts developed using solid-state NMR spectroscopy 53,59 . Notably, these structures represent snapshots of the kinetic cores of aggregation or end-state morphologies of amyloid fibrils and therefore provide limited information on the underlying aggregation pathways and toxicity-related effects of amyloids. On the other hand, the growing number of highresolution cryo-EM structures has highlighted the in vivo structural diversity of amyloid fibrils ${ }^{60}$, whereas steric zippers have been recently used for the development of targeted therapeutics ${ }^{61-63}$. However, determining the structural layout of amyloid fibrils still remains challenging. Cordax attempts to provide a cost-effective complementary powerful computational alternative that can be operated without any required scientific expertise necessary to apply the intricate technical approaches. Apart from its function as an aggregation predictor, the tool is uniquely poised to provide detailed complementary structural information on the putative amyloid fibril architecture of identified APRs. Users can utilise the method to structurally characterise identified APRs by classifying their overall specific topological preferences, including $\beta$-strand directionality and key residue positions that are integral parts of the amyloid core. The latter information is imperative for efforts focused on understanding the underlying mechanisms that dictate amyloid-related diseases or the formation of functional amyloids, but can also have an immense impact on the design of applied nanobiomaterials ${ }^{64}$, targeted amyloid inducers ${ }^{65}$ or counteragents, following the increased interest in the development of structurebased inhibitors of aggregation ${ }^{61-63}$.

\section{Methods}

Regression model training. In previous work we synthesised and explored the aggregation potential of 940 peptide sequences derived from both functional and pathological amyloid-forming proteins, which were supplemented with additional data on 462 hexapeptides derived from other published sources to develop WALTZ-DB $2.0^{32}$, the largest public comprehensive repository of experimentally defined amyloidogenic peptides. In total, 1402 hexapeptide sequences from WALTZ-DB were modelled on the 140 backbone structures of the Cordax library, leading to the generation of 196,280 models. The thermodynamic stability of each model $\left(\Delta G, \mathrm{kcal} \mathrm{mol}^{-1}\right)$ was calculated using FoldX and fed into a logistic regression model (Fig. 2c). This model was used to distil the aggregation propensity from the free energy values. Towards this end, from the calculated $\Delta G$ s, we isolated 50 representative energies using a recursive feature elimination algorithm (using the RFE module of the SciKit-learn python package ${ }^{33}$ and selecting for the set of templates that maximised the AUC). As a result, each sequence is described with a 50-dimensional vector. Next, the data were transformed in order to be constrained in a scoring range between 0 and 1 , using a Min/Max scaling algorithm. The regression model was trained with $\mathrm{L} 2$ penality and regularisation strength $(C)$ equal to 1 . Both scaling of the estimated $\Delta G$ and the machine-learning model were developed using the SciKit-learn python package ${ }^{66}$.

Model pipeline. Cordax receives a protein sequence in FASTA format as input, which is fragmented into hexapeptides using a sliding window process. Sequences are then threaded against the fragment library utilising FoldX and the derived free energies are translated into scoring values for every peptide window. An energetically fitted model is selected as the closest representative of the overall topology of the amyloid fibril core for each predicted window and is provided as output in standard PDB format to the users (Fig. 2c). An amyloidogenic profile is generated by scoring every single residue of the input sequence with the maximum calculated score of the corresponding windows, followed by a binary prediction for every segment. Finally, calculated energies are stored automatically in a growing local database and can be retrieved, thus creating a 'lazy' interface that bypasses unnecessary computation for recurring sequence segments or future runs.

Datasets. Performance assessment of Cordax was carried out utilising two individual data sets for peptide and protein aggregation propensity detection. Further validation of the method was performed against an independent subset screen of 96 hexapeptides sequences.

For peptide aggregation propensity, we used a dataset of 1402 non-redundant hexapeptides contained in the WALTZ-DB 2.0 repository ${ }^{32}$. This database is the 
largest currently available resource of experimentally characterised amyloidogenic peptides. It contains annotated peptide entries that are distributed in shorter subsets and extracted from literature $22,23,67-69$, in addition to peptides with experimentally determined amyloid-forming properties. As a result, it has been widely used as a validation set for several aggregation predicting tools $21,23,67,70,71$.

Collected in 2013, reg33 is a standard dataset for estimating the performance of aggregation propensity prediction in protein sequences ${ }^{25}$. It contains regional annotation of aggregating segments identified for 34 well-known amyloidogenic proteins. The annotation is assigned on a residue basis, thus containing 1260 residues in defined APRs and 6472 residues located in non-aggregating segments.

Last, we compiled a set consisting of 96 hexapeptide segments derived from potentially mis-annotated non-amyloidogenic regions of the reg33 dataset that were predicted as aggregation-prone segments after applying Cordax. Peptide segments were filtered for potential overlaps to the WALTZ-DB 2.0 set (Supplementary Data 2)

Comparative analysis. Binary classification was utilised to determine performances of calculated aggregation propensities per hexapeptide fragment or per residue. As a result, predictions can be classified by comparison to experimental validation into true positives (TP), true negatives (TN), false positives (FP) and false negatives (FN), respectively. Performance is evaluated using the following metrics:

$$
\begin{gathered}
\text { Accuracy }=\frac{\mathrm{TP}+\mathrm{TN}}{\mathrm{TP}+\mathrm{TN}+\mathrm{FP}+\mathrm{FN}} \\
\text { Precision }=\frac{\mathrm{TP}}{\mathrm{TP}+\mathrm{FP}} \\
\text { Sensitivity }(\text { Recall })=\frac{\mathrm{TP}}{\mathrm{TP}+\mathrm{FN}} \\
\text { Specificity }=\frac{\mathrm{TN}}{\mathrm{TN}+\mathrm{FP}} \\
\mathrm{F} 1=2 \times \frac{(\text { Precision } \times \text { Recall })}{(\text { Precision }+ \text { Recall })} \\
\mathrm{MCC}=\frac{(\mathrm{TP} \times \mathrm{TN}-\mathrm{FP} \times \mathrm{FN})}{\sqrt{(\mathrm{TN}+\mathrm{FN})(\mathrm{TN}+\mathrm{FP})(\mathrm{TP}+\mathrm{FN})(\mathrm{TP}+\mathrm{FP})}}
\end{gathered}
$$

Peptide synthesis. Peptides derived from the Cordax validation set were synthesised using an Intavis Multipep RSi solid phase peptide synthesis robot. Peptide purity $(>90 \%)$ was evaluated using RP-HPLC purification protocols and peptides were stored as ether precipitates $\left(-20^{\circ} \mathrm{C}\right)$. Peptide stocks were initially treated with 1,1,1,3,3,3-hexafluoro-isopropanol (HFIP) (Merck), then dissolved in traces of dimethyl sulfoxide (DMSO) (Merck) $(<5 \%)$, filtered through $0.2 \mu \mathrm{m}$ filters and finally in milli-Q water to reach a final concentration of $200 \mu \mathrm{M}$ or up to $1 \mathrm{mM}$ for dye-negative peptides. Dithiothreitol (DTT) $(1 \mathrm{mM})$ was included in solutions of peptides spanning cysteine or methionine residues. All peptides were incubated at room temperature for a period of 5 days on a rotating wheel.

Thioflavin-T and pFTAA-binding assays. Amyloid aggregation was monitored using fluorescent spectroscopy-binding assays. Th-T (Sigma) or pFTAA (Ebba Biotech $\mathrm{AB}$ ) was added in half-area black 96-well microplates (Corning, USA) at a final concentration of 25 and $0.5 \mu \mathrm{M}$, respectively. Fluorescence intensity was measured in replicates $(n=6)$ using a PolarStar Optima and a FluoStar Omega plate reader (BMG Labtech, Germany), equipped with an excitation filter at $440 \mathrm{~nm}$ and emission filters at 490 and $510 \mathrm{~nm}$, respectively.

Transmission electron microscopy. Peptide solutions were incubated for 5 days at room temperature in order to form mature amyloid-like fibrils. Suspensions (5 $\mu \mathrm{L}$ ) of each peptide solution were added on 400-mesh carbon-coated copper grids (Agar Scientific Ltd., England), following a glow-discharging step of $30 \mathrm{~s}$ to improve sample adsorption. Grids were washed with milli- $Q$ water and negatively stained using uranyl acetate ( $2 \% \mathrm{w} / \mathrm{v}$ in milli-Q water). Grids were examined with a JEM-1400 $120 \mathrm{kV}$ transmission electron microscope (JEOL, Japan), operated at 80 $\mathrm{keV}$.

Congo red staining. Droplets $(10 \mu \mathrm{L})$ of peptide solutions containing mature amyloid fibrils were cast on glass slides and permitted to dry slowly in ambient conditions in order to form thin films. The films were stained with a Congo red (Sigma) solution $(0.1 \% \mathrm{w} / \mathrm{v})$ prepared in milli-Q water for $20 \mathrm{~min}$. De-staining was performed with gradient ethanol solutions (70-90\%).

Determination of peptide propensities. Surface exposure and secondary structure analysis was performed using the FoldX energy force field on the available crystal structures for acylphosphatase-2 (PDB ID:1APS), amphoterin (PDB
ID:1CKT and 1HME), apolipoprotein-C2 (PDB ID:115J), $\alpha$-synuclein (PDB ID:1XQ8), $\beta 2$-microglobulin (PDB ID:1A1M), casein (PDB ID:6FS5), gelsolin (PDB ID:3FFN), Het-S (PDB ID:2WVN), kerato-epithelin (PDB ID:5NV6), lactoferrin (PDB ID:1CB6), prolactin (PDB ID:1RW5), major prion protein (PDB ID:1E1G), repA (PDB ID:1HKQ), serum amyloid alpha (PDB ID:4IP8), Sup35 (PDB ID:4CRN) and Ure2p (PDB ID:1HQO). Partition coefficients were calculated using $\mathrm{PlogP}$, which specialises in peptides with blocked termini ${ }^{72}$. Structural alignment and visualisation were performed with the aid of YASARA ${ }^{73}$. Sequence similarities were calculated using the BLOSUM62 matrix currently available under the Biostrings R library. Correlation plots were generated using the ggpairs() function available under the GGally R library and ROC curves were calculated using ROCR.

Dimensionality reduction analysis. A defined amyloid-forming sequence space was constructed by merging the experimentally determined amyloid sequences of the 96-peptide screen, identified by Cordax, to the amyloid sequence content extracted from WALTZ-DB. Prior to t-SNE analysis, scoring outputs using Cordax, PASTA $^{23}$, TANGO ${ }^{7}$ and WALTZ ${ }^{21}$ were calculated for each peptide entry. Peptide description was complemented with a 20 -dimensional vector using the available $\mathrm{R}$ package Peptides. All data points were reduced and embedded in 2D-space using the Rtsne package, with perplexity $(p=45)$, iteration steps $(n=5000)$ and learning rate (default) defined based on the initial guidelines proposed by van der Maaten and Hinton ${ }^{74}$. UMAP reduction was performed using the R umap package and three-dimensional PCA analysis was conducted using pca3d R package and visualised with scatter3D, respectively.

Reporting summary. Further information on research design is available in the Nature Research Reporting Summary linked to this article.

\section{Data availability}

The source data underlying Figs. $3 \mathrm{a}-\mathrm{d}, 4 \mathrm{l}, \mathrm{m}$ and $6 \mathrm{a}, \mathrm{c}, \mathrm{d}$ are provided as a Source Data file. Other data are available from the corresponding authors upon reasonable request.

\section{Code availability}

CORDAX is implemented in Python and is freely accessible for academic and non-profit users at https://cordax.switchlab.org/.

Received: 9 March 2020; Accepted: 18 June 2020; Published online: 03 July 2020

\section{References}

1. Benson, M. D. et al. Amyloid nomenclature 2018: recommendations by the International Society of Amyloidosis (ISA) nomenclature committee. Amyloid 25, 215-219 (2018).

2. Chiti, F. \& Dobson, C. M. Protein misfolding, amyloid formation, and human disease: a summary of progress over the last decade. Annu. Rev. Biochem. 86, 27-68 (2017).

3. Pham, C. L., Kwan, A. H. \& Sunde, M. Functional amyloid: widespread in nature, diverse in purpose. Essays Biochem. 56, 207-219 (2014).

4. Stefani, M. \& Dobson, C. M. Protein aggregation and aggregate toxicity: new insights into protein folding, misfolding diseases and biological evolution. $J$. Mol. Med. 81, 678-699 (2003).

5. Lopez de la Paz, M. \& Serrano, L. Sequence determinants of amyloid fibril formation. Proc. Natl Acad. Sci. USA 101, 87-92 (2004).

6. Chiti, F., Stefani, M., Taddei, N., Ramponi, G. \& Dobson, C. M. Rationalization of the effects of mutations on peptide and protein aggregation rates. Nature 424, 805-808 (2003).

7. Fernandez-Escamilla, A. M., Rousseau, F., Schymkowitz, J. \& Serrano, L. Prediction of sequence-dependent and mutational effects on the aggregation of peptides and proteins. Nat. Biotechnol. 22, 1302-1306 (2004).

8. Pawar, A. P. et al. Prediction of "aggregation-prone" and "aggregationsusceptible" regions in proteins associated with neurodegenerative diseases. $J$. Mol. Biol. 350, 379-392 (2005).

9. de Groot, N. S., Castillo, V., Grana-Montes, R. \& Ventura, S. AGGRESCAN: method, application, and perspectives for drug design. Methods Mol. Biol. 819, 199-220 (2012).

10. Tartaglia, G. G. et al. Prediction of aggregation-prone regions in structured proteins. J. Mol. Biol. 380, 425-436 (2008).

11. Beerten, J., Schymkowitz, J. \& Rousseau, F. Aggregation prone regions and gatekeeping residues in protein sequences. Curr. Top. Med. Chem. 12, 2470-2478 (2012)

12. Buck, P. M., Kumar, S. \& Singh, S. K. On the role of aggregation prone regions in protein evolution, stability, and enzymatic catalysis: insights from diverse analyses. PLoS Comput. Biol. 9, e1003291 (2013). 
13. Castillo, V. \& Ventura, S. Amyloidogenic regions and interaction surfaces overlap in globular proteins related to conformational diseases. PLoS Comput. Biol. 5, e1000476 (2009).

14. Dobson, C. M. Protein folding and misfolding. Nature 426, 884-890 (2003).

15. Mishra, A., Ranganathan, S., Jayaram, B. \& Sattar, A. Role of solvent accessibility for aggregation-prone patches in protein folding. Sci. Rep. 8, 12896 (2018).

16. Alberti, S., Gladfelter, A. \& Mittag, T. Considerations and challenges in studying liquid-liquid phase separation and biomolecular condensates. Cell 176, 419-434 (2019).

17. Mohammadi, P. et al. Phase transitions as intermediate steps in the formation of molecularly engineered protein fibers. Commun. Biol. 1, 86 (2018).

18. Schmidt, H. B., Barreau, A. \& Rohatgi, R. Phase separation-deficient TDP43 remains functional in splicing. Nat. Commun. 10, 4890 (2019).

19. Hamodrakas, S. J. Protein aggregation and amyloid fibril formation prediction software from primary sequence: towards controlling the formation of bacterial inclusion bodies. FEBS J. 278, 2428-2435 (2011).

20. Gasior, P. \& Kotulska, M. FISH Amyloid-a new method for finding amyloidogenic segments in proteins based on site specific co-occurrence of aminoacids. BMC Bioinforma. 15, 54 (2014).

21. Maurer-Stroh, S. et al. Exploring the sequence determinants of amyloid structure using position-specific scoring matrices. Nat. Methods 7, 237-242 (2010).

22. Thangakani, A. M., Kumar, S., Nagarajan, R., Velmurugan, D. \& Gromiha, M. M. GAP: towards almost 100 percent prediction for beta-strand-mediated aggregating peptides with distinct morphologies. Bioinformatics 30, 1983-1990 (2014).

23. Walsh, I., Seno, F., Tosatto, S. C. \& Trovato, A. PASTA 2.0: an improved server for protein aggregation prediction. Nucleic Acids Res. 42, W301-W307 (2014).

24. Emily, M., Talvas, A. \& Delamarche, C. MetAmyl: a METa-predictor for AMYLoid proteins. PLoS ONE 8, e79722 (2013).

25. Tsolis, A. C., Papandreou, N. C., Iconomidou, V. A. \& Hamodrakas, S. J. A consensus method for the prediction of 'aggregation-prone' peptides in globular proteins. PLoS ONE 8, e54175 (2013).

26. Kim, C., Choi, J., Lee, S. J., Welsh, W. J. \& Yoon, S. NetCSSP: web application for predicting chameleon sequences and amyloid fibril formation. Nucleic Acids Res. 37, W469-W473 (2009)

27. Yoon, S. \& Welsh, W. J. Detecting hidden sequence propensity for amyloid fibril formation. Protein Sci. 13, 2149-2160 (2004).

28. Bondarev, S. A., Bondareva, O. V., Zhouravleva, G. A. \& Kajava, A. V. BetaSerpentine: a bioinformatics tool for reconstruction of amyloid structures. Bioinformatics 34, 599-608 (2018).

29. Thompson, M. J. et al. The 3D profile method for identifying fibril-forming segments of proteins. Proc. Natl Acad. Sci. USA 103, 4074-4078 (2006)

30. Schymkowitz, J. et al. The FoldX web server: an online force field. Nucleic acids Res. 33, W382-W388 (2005).

31. Sawaya, M. R. et al. Atomic structures of amyloid cross-beta spines reveal varied steric zippers. Nature 447, 453-457 (2007).

32. Louros, N. et al. WALTZ-DB 2.0: an updated database containing structural information of experimentally determined amyloid-forming peptides. Nucleic Acids Res. https://doi.org/10.1093/nar/gkz758 (2019).

33. Sing, T., Sander, O., Beerenwinkel, N. \& Lengauer, T. ROCR: visualizing classifier performance in R. Bioinformatics 21, 3940-3941 (2005).

34. Munir, F., Gull, S., Asif, A. \& Minhas, F. MILAMP: multiple instance prediction of amyloid proteins. IEEE/ACM Trans. Comput. Biol. Bioinform. https://doi.org/10.1109/TCBB.2019.2936846 (2019).

35. Iconomidou, V. A., Leontis, A., Hoenger, A. \& Hamodrakas, S. J. Identification of a novel 'aggregation-prone'/'amyloidogenic determinant' peptide in the sequence of the highly amyloidogenic human calcitonin. FEBS Lett. 587, 569-574 (2013).

36. Tsiolaki, P. L., Louros, N. N., Hamodrakas, S. J. \& Iconomidou, V. A Exploring the 'aggregation-prone' core of human Cystatin C: a structural study. J. Struct. Biol. 191, 272-280 (2015).

37. Saelices, L. et al. Uncovering the mechanism of aggregation of human transthyretin. J. Biol. Chem. 290, 28932-28943 (2015).

38. Baxa, U. et al. Characterization of beta-sheet structure in Ure2p1-89 yeast prion fibrils by solid-state nuclear magnetic resonance. Biochemistry $\mathbf{4 6}$, 13149-13162 (2007).

39. Gross, M. et al. Formation of amyloid fibrils by peptides derived from the bacterial cold shock protein CspB. Protein Sci. 8, 1350-1357 (1999).

40. Louros, N. N. et al. Chameleon 'aggregation-prone' segments of apoA-I: A model of amyloid fibrils formed in apoA-I amyloidosis. Int. J. Biol. Macromol. 79, 711-718 (2015)

41. Van Melckebeke, H. et al. Atomic-resolution three-dimensional structure of HET-s(218-289) amyloid fibrils by solid-state NMR spectroscopy. J. Am. Chem. Soc. 132, 13765-13775 (2010).

42. Rauscher, S., Baud, S., Miao, M., Keeley, F. W. \& Pomes, R. Proline and glycine control protein self-organization into elastomeric or amyloid fibrils. Structure 14, 1667-1676 (2006).
43. Tsiolaki, P. L., Louros, N. N. \& Iconomidou, V. A. Hexapeptide tandem repeats dictate the formation of silkmoth chorion, a natural protective amyloid. J. Mol. Biol. 430, 3774-3783 (2018).

44. Chernoff, Y. O. Amyloidogenic domains, prions and structural inheritance: rudiments of early life or recent acquisition? Curr. Opin. Chem. Biol. 8, 665-671 (2004).

45. Greenwald, J., Friedmann, M. P. \& Riek, R. Amyloid aggregates arise from amino acid condensations under prebiotic conditions. Angew. Chem. 55, 11609-11613 (2016)

46. Martin, E. W. \& Mittag, T. Relationship of sequence and phase separation in protein low-complexity regions. Biochemistry 57, 2478-2487 (2018).

47. Rousseau, F., Serrano, L. \& Schymkowitz, J. W. How evolutionary pressure against protein aggregation shaped chaperone specificity. J. Mol. Biol. 355, 1037-1047 (2006)

48. Gazit, E. Self assembly of short aromatic peptides into amyloid fibrils and related nanostructures. Prion 1, 32-35 (2007).

49. Tabatabaei Ghomi, H., Topp, E. M. \& Lill, M. A. Fibpredictor: a computational method for rapid prediction of amyloid fibril structures. J. Mol. Model. 22, 206 (2016)

50. Landau, M. et al. Towards a pharmacophore for amyloid. PLoS Biol. 9, e1001080 (2011).

51. Berhanu, W. M. \& Masunov, A. E. Alternative packing modes leading to amyloid polymorphism in five fragments studied with molecular dynamics. Biopolymers 98, 131-144 (2012).

52. Yu, L., Lee, S. J. \& Yee, V. C. Crystal structures of polymorphic prion protein betal peptides reveal variable steric zipper conformations. Biochemistry 54, 3640-3648 (2015)

53. Tycko, R. Amyloid polymorphism: structural basis and neurobiological relevance. Neuron 86, 632-645 (2015).

54. Close, W. et al. Physical basis of amyloid fibril polymorphism. Nat. Commun. 9, 699 (2018)

55. Perov, S. et al. Structural insights into curli CsgA cross-beta fibril architecture inspire repurposing of anti-amyloid compounds as anti-biofilm agents. PLoS Pathog. 15, e1007978 (2019).

56. Do, T. D. et al. Distal amyloid beta-protein fragments template amyloid assembly. Protein Sci. 27, 1181-1190 (2018).

57. Nannenga, B. L. \& Gonen, T. The cryo-EM method microcrystal electron diffraction (MicroED). Nat. Methods 16, 369-379 (2019).

58. Fandrich, M. et al. Amyloid fibril polymorphism: a challenge for molecular imaging and therapy. J. Intern. Med. 283, 218-237 (2018).

59. Tycko, R. Molecular structure of aggregated amyloid-beta: insights from solidstate nuclear magnetic resonance. Cold Spring Harbor Perspect. Med. 6, https://doi.org/10.1101/cshperspect.a024083 (2016).

60. Gallardo, R., Ranson, N. A. \& Radford, S. E. Amyloid structures: much more than just a cross-beta fold. Curr. Opin. Struct. Biol. 60, 7-16 (2020)

61. Lu, J. et al. Structure-based peptide inhibitor design of amyloid-beta aggregation. Front. Mol. Neurosci. 12, 54 (2019).

62. Seidler, P. M. et al. Structure-based inhibitors halt prion-like seeding by Alzheimer's disease- and tauopathy-derived brain tissue samples. J. Biol. Chem. https://doi.org/10.1074/jbc.RA119.009688 (2019).

63. Sivanesam, K. et al. Peptide Inhibitors of the amyloidogenesis of IAPP: verification of the hairpin-binding geometry hypothesis. FEBS Lett. 590, 2575-2583 (2016).

64. Mitraki, A. Protein aggregation from inclusion bodies to amyloid and biomaterials. Adv. Protein Chem. Struct. Biol. 79, 89-125 (2010).

65. Khodaparast, L. et al. Aggregating sequences that occur in many proteins constitute weak spots of bacterial proteostasis. Nat. Commun. 9, 866 (2018).

66. Pedegrosa, F. et al. Scikit-learn: machine learning in python. JMLR 12, 2825-2830 (2011).

67. Chen, M., Schafer, N. P., Zheng, W. \& Wolynes, P. G. The Associative Memory, Water Mediated, Structure and Energy Model (AWSEM)Amylometer: predicting amyloid propensity and fibril topology using an optimized folding landscape model. ACS Chem. Neurosci. 9, 1027-1039 (2018).

68. Varadi, M., De Baets, G., Vranken, W. F., Tompa, P. \& Pancsa, R. AmyPro: a database of proteins with validated amyloidogenic regions. Nucleic Acids Res. 46, D387-D392 (2018).

69. Wozniak, P. P. \& Kotulska, M. AmyLoad: website dedicated to amyloidogenic protein fragments. Bioinformatics 31, 3395-3397 (2015).

70. Niu, M., Li, Y., Wang, C. \& Han, K. RFAmyloid: a web server for predicting amyloid proteins. Int. J. Mol. Sci. 19, https://doi.org/10.3390/ijms19072071 (2018).

71. Sankar, K., Krystek, S. R. Jr., Carl, S. M., Day, T. \& Maier, J. K. X. AggScore: prediction of aggregation-prone regions in proteins based on the distribution of surface patches. Proteins 86, 1147-1156 (2018).

72. Tao, P., Wang, R. \& Lai, L. Calculating partition coefficients of peptides by the addition method. Mol. Model. Annu. 5, 189-195 (1999).

73. Krieger, E. \& Vriend, G. YASARA View—molecular graphics for all devicesfrom smartphones to workstations. Bioinformatics 30, 2981-2982 (2014). 
74. van der Maaten, L. J. P. \& Hinton, G. E. Visualizing high-dimensional data using t-SNE. J. Mach. Learn. Res. 9, 2579-2605 (2008).

\section{Acknowledgements}

This work was supported by Flanders institute for Biotechnology (VIB); the University of Leuven; the Fund for Scientific Research Flanders (FWO, Hercules Foundation grant AKUL/15/34-G0H1716N and project grant G0C2818N); European Research Council under the European Union's Horizon 2020 Framework Programme ERC Grant Agreement [647458 (MANGO) to J.S.]; N.L. was funded by Fund for Scientific Research Flanders Post-doctoral Fellowship (FWO) [12P0919N to N.L.]. All electron microscopy imagings were performed at the Electron Microscopy Platform of VIB Bio Imaging Core at KU Leuven.

\section{Author contributions}

N.L., F.R., and J.S. conceived and designed the study. N.L. and M.D.V. designed and synthesised the peptide library. G.O. contributed to the development of the logistic regression model. N.L. performed the in silico analysis, the experimental work and analysed the data. F.R. and J.S. supervised the project. N.L., F.R. and J.S. prepared the manuscript

\section{Competing interests}

The host institute VIB filed a patent application with the European patent office (EP 20176563.3) entitled "MEANS AND METHODS FOR THE PREDICTION OF AMYLOID CORE SEQUENCES” which mentions the authors (N.L., G.O., J.S. and F.R.) as inventors. This patent describes the Cordax method covered in this manuscript.

\section{Additional information}

Supplementary information is available for this paper at https://doi.org/10.1038/s41467020-17207-3.

Correspondence and requests for materials should be addressed to F.R. or J.S.

Peer review information Nature Communications thanks Gian Gaetano Tartaglia and the other, anonymous, reviewer(s) for their contribution to the peer review of this work

Reprints and permission information is available at http://www.nature.com/reprints

Publisher's note Springer Nature remains neutral with regard to jurisdictional claims in published maps and institutional affiliations.

(c) (i) Open Access This article is licensed under a Creative Commons Attribution 4.0 International License, which permits use, sharing, adaptation, distribution and reproduction in any medium or format, as long as you give appropriate credit to the original author(s) and the source, provide a link to the Creative Commons license, and indicate if changes were made. The images or other third party material in this article are included in the article's Creative Commons license, unless indicated otherwise in a credit line to the material. If material is not included in the article's Creative Commons license and your intended use is not permitted by statutory regulation or exceeds the permitted use, you will need to obtain permission directly from the copyright holder. To view a copy of this license, visit http://creativecommons.org/ licenses/by/4.0/.

(C) The Author(s) 2020 